\title{
Echinacoside promotes the proliferation of human renal tubular epithelial cells by blocking the HBX/TREM2-mediated NF-кB signalling pathway
}

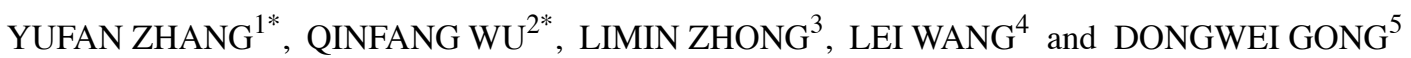 \\ Departments of ${ }^{1}$ Traditional Chinese Medicine, ${ }^{2}$ Paediatrics, ${ }^{3}$ Pharmacy, ${ }^{4}$ Rehabilitation and ${ }^{5}$ Surgery, \\ Yangpu District Kongjiang Hospital, Shanghai 200093, P.R. China
}

Received August 8, 2019; Accepted March 26, 2020

DOI: $10.3892 / \mathrm{mmr} .2020 .11201$

\begin{abstract}
Hepatitis B virus X (HBX) protein is required for the replication of HBV and plays a role in the progression of hepatitis in humans. However, the underlying function of HBX during HBV-induced chronic glomerulonephritis (HBV-GN) is unknown. Echinacoside (ECH) is a phenylethanoid glycoside from the Cistanche genus, which possesses strong antiapoptosis and neuroprotective activities. In the present study, the function of HBX and the relationship between HBX and ECH in human renal tubular epithelial cells (RTECs; HK-2 cell line) were explored. Reverse transcription-quantitative PCR and western blot analyses were used to quantify the mRNA and protein expression levels of HBX in HK-2 cells, respectively. The Cell Counting Kit- 8 assay was performed to analyse cell proliferation. Flow cytometry analysis was used to determine the rate of apoptosis. HBX showed antiproliferative and proapoptotic effects in HK-2 cells and was positively associated with triggering receptor expressed on myeloid cells 2 (TREM2) expression. Furthermore, ECH disrupted the function of HBX in HK-2 cells, functioning as an HBX suppressor. Moreover, a specific NF- $\mathrm{kB}$ inhibitor, PDTC, was used to further examine the relationship between HBX and $\mathrm{NF}-\kappa \mathrm{B}$. The results suggested that NF- $\mathrm{BB}$ was involved in the HBX/TREM2 signaling pathway and negatively regulated TREM2 expression in RTECs. The present study provided novel insights into the function of HBX, and also indicated the potential value of $\mathrm{ECH}$ as a therapeutic agent for HBV-GN.
\end{abstract}

Correspondence to: Dr Dongwei Gong, Department of Surgery, Yangpu District Kongjiang Hospital, 480 Shuangyang Road, Shanghai 200093, P.R. China

E-mail: m13917178534_2@163.com

*Contributed equally

Key words: hepatitis B virus X, HBV-GN, echinacoside, TREM2, $\mathrm{NF}-\kappa \mathrm{B}$

\section{Introduction}

Hepatitis B virus (HBV) is a Hepadnaviridae virus, which leads to acute and chronic hepatitis in humans (1). HBV, not only causes persistent or transient infection in the liver, but also infects extrahepatic tissue, including the pancreas, bile duct, lymphoid system and kidneys (2). Moreover, HBV infection is closely associated with chronic glomerulonephritis (HBV-GN); however, the molecular mechanisms of HBV during HBV-GN are not completely understood $(3,4)$.

HBV regulatory hepatitis B virus $\mathrm{X}(\mathrm{HBX})$ protein is required for HBV replication $(5,6)$. A number of studies have reported that HBX promotes cell proliferation during HBV infection-associated hepatocarcinoma (7-9). Human renal tubular epithelial cells (RTECs) are a major part of the renal tubular interstitium and play an active role in renal inflammation (10). RTEC apoptosis is closely associated with the dysfunction of the tubular interstitium, which subsequently leads to the progression of HBV-GN (10). HBX is primarily expressed in RTEC cells and causes RTEC apoptosis and renal tube lesions in patients with HBV-GN $(11,12)$. Furthermore, HBX has been reported to suppress the proliferation of rat RTECs (13), and HBX overexpression accelerates the cell cycle progression from $\mathrm{G}_{1}$ to $S$ phase in primary RTECs, leading to cell cycle arrest in the $S$ phase (14). Therefore, identifying a HBX inhibitor may provide a novel therapeutic for HBV-GN. However, the precise molecular network of HBX in human RTECs is not completely understood.

Echinacoside $(\mathrm{ECH})$ is a natural compound extracted from Cistanche plants, which shows antiapoptotic and anti-inflammatory effects $(15,16)$. A previous study reported that ECH induces the proliferation and prevents the apoptosis of intestinal epithelial cells (17). Furthermore, ECH has been reported to accelerate bone regeneration by increasing the proliferation of osteoblast cells (18). ECH can also decrease HBV replication, antigen expression and inflammation in rat intestine epithelial cells $(16,19)$. However, the biological effect of ECH on RTECs has not been fully elucidated.

Triggering receptor expressed on myeloid cells 2 (TREM2), a member of the transmembrane receptor family, plays a role in maintaining adequate microglial metabolism during Alzheimer's disease $(20,21)$. A previous study indicated that 
TREM2 overexpression promoted the proliferation of glioma cells (22). By contrast, TREM2 knockdown decreases the translocation of NF- $\mathrm{KB}$ to the nucleus in degenerative human nucleus pulposus cells (23). Furthermore, TREM2 has been identified as a novel therapeutic target for human intervertebral disc degeneration (23). However, the biological function of TREM2 in human RTECs has not been reported.

In the present study, HBX overexpression (oeHBX) was induced in human RTECs (HK-2 cell line) and subsequently, the effect of ECH on transfected oeHBX cells was investigated. The aim of the present study was to investigate the functions of HBX and the potential value of $\mathrm{ECH}$ as an effective therapeutic agent for HBV-GN.

\section{Materials and methods}

Cell culture. The human RTEC cell line HK-2 was purchased from Shanghai Yaji Biotechnology Co., Ltd. HK-2 cells were cultured in DMEM/F12 media (cat. no. SH30023.01B; HyClone; Cytiva) supplemented with $10 \%$ foetal bovine serum (cat. no. 16000-044; Gibco; Thermo Fisher Scientific, Inc.), $2 \mathrm{mM} \mathrm{L-glutamine}$ and $1 \%$ penicillin/streptomycin (cat. no. P1400-100; Beijing Solarbio Science \& Technology Co., Ltd.) at $37^{\circ} \mathrm{C}$ with $5 \% \mathrm{CO}_{2}$.

$R N A$ isolation and reverse transcription-quantitative PCR $(R T-q P C R)$. Total RNA was extracted from HK-2 cells using TRIzol $^{\circledR}$ (cat. no. 1596-026; Invitrogen; Thermo Fisher Scientific, Inc.), according to the manufacturer's protocol. Total RNA was reverse transcribed into cDNA using the First Strand cDNA Synthesis kit according to the manufacturer's protocol (cat. no. K1622; Thermo Fisher Scientific, Inc.). qPCR was performed using SYBR-Green premix according to the manufacturer's protocol (cat. no. K0223; Thermo Fisher Scientific, Inc.)on an ABI-7300 real-time detector (Applied Biosystems; Thermo Fisher Scientific, Inc.). The conditions of PCR were: $95^{\circ} \mathrm{C}$ for $10 \mathrm{~min}$ followed by 40 cycles of $95^{\circ} \mathrm{C}$ for $15 \mathrm{sec}, 60^{\circ} \mathrm{C}$ for $45 \mathrm{sec}$. Three repeats were needed for each reaction. The following primer pairs were used for the qPCR: HBX forward, 5'-GGCTGCTAGGTTGTACTG-3' and reverse, 5'-CAGAGGTGAAGCGAAGTG-3'; TREM2 forward, 5'-TGGCACTCTCACCATTACG-3' and reverse, 5'-CCTCCC ATCATCTTCCTTCAC-3'; and GAPDH forward, 5'-AAT CCCATCACCATCTTC-3' and reverse, 5'-AGGCTGTTG TCATACTTC-3'. mRNA levels were quantified using the $2^{-\triangle \Delta C q}$ method and normalized to the internal reference gene GAPDH (24).

Overexpression and knockdown. To induce the overexpression of HBX and TREM2, the full-length HBX or TREM2 cDNA sequences were inserted into the pLVX-puro vector (Clontech Laboratories, Inc.) by double digestion (HindIII and EcoRI). Subsequently, the recombined plasmid $(1.5 \mu \mathrm{g})$ was transfected into HK-2 cells $\left(2 \times 10^{5}\right)$. The mock plasmid (an empty vector, $1 \mu \mathrm{g})$ was transiently transfected into HK-2 cells $\left(2 \times 10^{5}\right)$ as a negative control (oeNC) using Lipofectamine ${ }^{\circledR} 2000$ (cat. no. 11668-027, Invitrogen, Thermo Fisher Scientific, Inc.). ECH (cat. no. 82854-37-3; Biopurify Phytochemicals, Ltd.) was dissolved in DMSO (cat. no. D2650; Sigma-Aldrich; Merck KGaA) at the concentration of 1, 2.5, 5, 10, 20 and
$50 \mathrm{mg} / \mathrm{l}$ respectively; and added to the culture of transfected oeHBX or oeTREM 2 cells. The subsequent experimentation began $48 \mathrm{~h}$ later.

To knock down TREM 2 expression, three small interfering (si)RNAs (siTREM2-1, siTREM2-2 and siTREM2-3; $20 \mu \mathrm{M}$ ) targeting TREM2 were synthesized (Shanghai Majorbio Bio-Pharm Technology Co. Ltd.) and subsequently transfected into HK-2 cells $\left(2 \times 10^{5}\right)$ using Lipofectamine ${ }^{\circledR} 2000$ according to the manufacturer's protocol (Invitrogen; Thermo Fisher Scientific, Inc.). A non-specific scrambled siRNA sequence (5'UUCUCCGAACGUGUCACGU3, $25 \mathrm{nM}$ ) si-negative control (NC) was used as the negative control. The targeted locus and sequences of the TREM 2 siRNAs are provided in Table SI. The subsequent experimentation was performed $48 \mathrm{~h}$ later.

Western blotting. Total protein was extracted from HK-2 cells using RIPA lysis buffer (cat. no. BYL40825; JRDUN Biotech Co., Ltd.). Total protein was quantified using the Bicinchoninic Acid assay kit (cat. no. PICPI23223; Thermo Fisher Scientific, Inc.) according to the manufacturer's protocol and $20 \mu \mathrm{g}$ protein/lane was separated via SDS-PAGE on a $15 \%$ gel. The densitometry of samples were determined using a microplate reader (DNM-9602, Pulangxin). Subsequently, the separated proteins were transferred onto a PVDF membrane. Following blocking with 5\% non-fat dry milk for $1 \mathrm{~h}$ at room temperature, the membrane was incubated with primary antibodies (Table SII) at $4^{\circ} \mathrm{C}$ overnight and re-incubated with the secondary antibody anti-mouse $\operatorname{IgG}(1: 1,000$, cat. no. A0216, Beyotime Institute of Biotechnology) for $1 \mathrm{~h}$ at $37^{\circ} \mathrm{C}$. The immunoreactive protein bands were visualized using an ECL system (Cytiva). Blots were performed in triplicate. GAPDH and $\mathrm{H} 3$ were used as the loading controls for cytoplasmic and nuclear proteins, respectively.

Cell proliferation. The Cell Counting Kit-8 (CCK-8; cat. no. CP002; SAB Biotherapeutics, Inc.)assay was performed to quantify cell proliferation. Briefly, a total of $3 \times 10^{4}$ cells/well were seeded into 96-well plates and CCK-8 reagent was added, according to the manufacturer's protocol. Subsequently, the optical density (OD) 450 value of each well was determined by a microplate reader at $0,12,24$ and $48 \mathrm{~h}$. The assay was performed in triplicate.

Cell apoptosis. Briefly, an Annexin V-FITC Apoptosis Detection kit (cat. no. C1063; Beyotime Institute of Biotechnology) was used to examine the number of apoptotic HK-2 cells, according to the manufacturer's protocol. After $48 \mathrm{~h}$ post-transfection, the cells were performed using a flow cytometer (Accuri C6, BD Biosciences) and analyzed using FlowJo software version 7.6.5 (FlowJo LLC). Three repeats were performed for each sample.

Luciferase reporter assay. The 3'-UTR sequence of TREM2 was inserted into the firefly luciferase reporter plasmid pGL3-Enhancer-luc2 (pGL3-Enhancer-luc2-pTREM2; E1910, Promega Corporation) by double digestion using XhoI and HindIII. Subsequently, the pGL3-Enhancer-luc2-pTREM2 plasmid $(1.5 \mu \mathrm{g})$ was transfected into oeHBX and oeNC cells $\left(2 \times 10^{5}\right)$ using Lipofectamine ${ }^{\circledR} 2000$ (cat. no. 11668-027, 
A

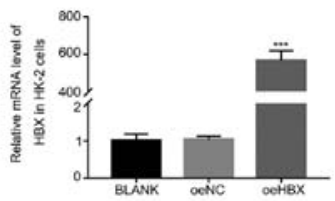

C

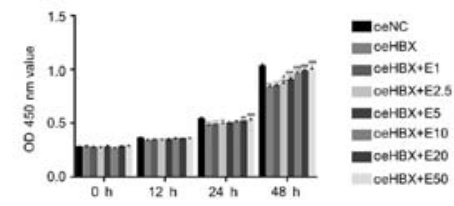

D
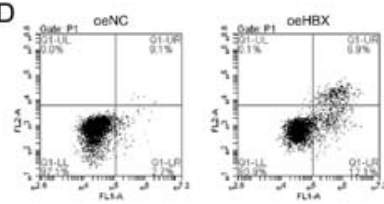

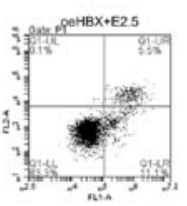

B
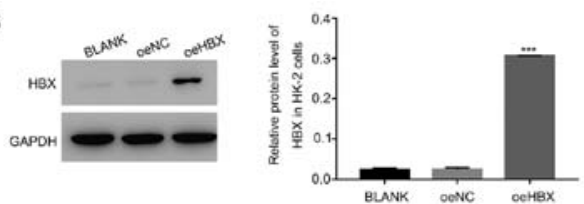

Figure 1. ECH suppresses the function of HBX in HK-2 cells. Relative (A) mRNA and (B) protein expression levels of HBX in HK-2 cells. (C) ECH increased the cell proliferation rate of oeHBX cells. (D) ECH reduced the apoptotic rate of oeHBX cells. ${ }^{*} \mathrm{P}<0.05,{ }^{* *} \mathrm{P}<0.01$ and ${ }^{* * * *} \mathrm{P}<0.001$ vs. oeNC. ${ }^{\#} \mathrm{P}<0.05,{ }^{\# \#} \mathrm{P}<0.01$

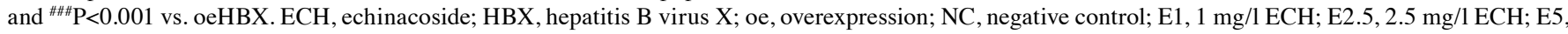
5 mg/1 ECH; E10, 10 mg/1 ECH; E20, 20 mg/1 ECH; E50, 50 mg/l ECH; OD, optical density.

Invitrogen, Thermo Fisher Scientific, Inc.). Additionally, the $\mathrm{NF}-\kappa \mathrm{B}$ inhibitor PDTC $(10 \mu \mathrm{M}$; cat. no. S1809; Beyotime Institute of Biotechnology) was dissolved in DMSO (cat. no. D2650; Sigma-Aldrich; Merck KGaA) and added to the oeHBX cell culture to knock down the expression of NF- $\mathrm{NB}$. Following incubation for $48 \mathrm{~h}$ at room temperature, the cells were collected. Subsequently, the firefly and Renilla luciferase activities were determined using the Dual-Luciferase Reporter Assay system (cat. no. E1910; Promega Corporation), according to the manufacturer's protocol. Firefly luciferase activity was normalized to Renilla luciferase activity. Three repeats were performed for each sample.

Statistical analysis. Statistical analyses were performed using GraphPad Prism software (version 7.0; GraphPad Software, Inc.). Data are presented as the mean \pm SD. Data were analysed using one-way ANOVA followed by Tukey's post hoc test. $\mathrm{P}<0.05$ was considered to indicate a statistically significant difference.

\section{Results}

ECH suppresses the function of $\mathrm{HBX}$ in $\mathrm{HK}-2$ cells. To assess the function of HBX, HBX overexpression was induced in HK-2 cells. The relative mRNA and protein levels of HBX were significantly upregulated in oeHBX cells compared with oeNC cells (Fig. 1A and B). Subsequently, oeHBX cells were cultured with different concentrations of ECH $(1,2.5,5,10$, 20 and $50 \mathrm{mg} / \mathrm{l}$; E1, E2.5, E5, E10, E20 and E50, respectively). HBX overexpression significantly reduced the proliferation of HK-2 cells, and this decrease was reversed by ECH in a dose-dependent manner at 24 and 48 h (Fig. 1C).

Furthermore, the proliferation rate of oeHBX cells displayed no significant difference compared with E1-treated cells (Fig. 1C). By contrast, E10 treatment significantly increased the proliferation rate of oeHBX cells compared with E20 and E50 treatment which exhibited no significant difference (Fig. 1C). Therefore, the effect of ECH on apoptosis was examined in E2.5, E5 and E10-treated cells. HBX overexpression significantly increased the apoptosis of HK-2 cells, and the apoptosis rate of oeHBX cells decreased with $\mathrm{ECH}$ treatment in a dose-dependent manner (Fig. 1D). Overall, the results suggested that $\mathrm{ECH}$ inhibited the function of $\mathrm{HBX}$ in HK-2 cells in a dose-dependent manner.

TREM2 expression is inhibited by ECH in transfected oeHBX cells. RT-qPCR and western blotting were used to examine the relative mRNA and protein expression levels of TREM2 in oeHBX cells, respectively. TREM 2 mRNA and protein expression levels were significantly upregulated in oeHBX cells compared with oeNC cells (Fig. 2A and B). However, $\mathrm{ECH}$ decreased the expression levels of TREM2 in transfected oeHBX cells in a dose-dependent manner (Fig. 2A and B). Therefore, the results suggested that HBX expression was positively associated with TREM2 expression, and further indicated the suppressive effect of ECH on HBX in HK-2 cells.

Knockdown and overexpression of TREM 2 in HK-2 cells. To further assess the function of TREM2, TREM2 knockdown and overexpression were induced in $\mathrm{HK}-2$ cells using RNA interference and a lentiviral vector, respectively. For the knockdown assay, three siRNAs targeting the human gene TREM2 (siTREM2-1, siTREM2-2 and siTREM2-3) were transfected into HK-2 cells, and a non-specific siRNA was used as a negative control (siNC). All three TREM2 siRNAs successfully knocked down the endogenous expression of TREM 2 in HK-2 cells (Fig. 3A and B). However, the lowest relative TREM2 mRNA and protein expression levels were observed in siTREM2-1 and siTREM2-2-transfected cells (Fig. 3A and B), therefore, siTREM2-1 and siTREM2-2-transfected cells were chosen for subsequent experimentation.

For the overexpression experiments, a plasmid containing the full-length human TREM2 cDNA sequence was constructed and subsequently transfected into HK-2 cells (oeTREM2). A 

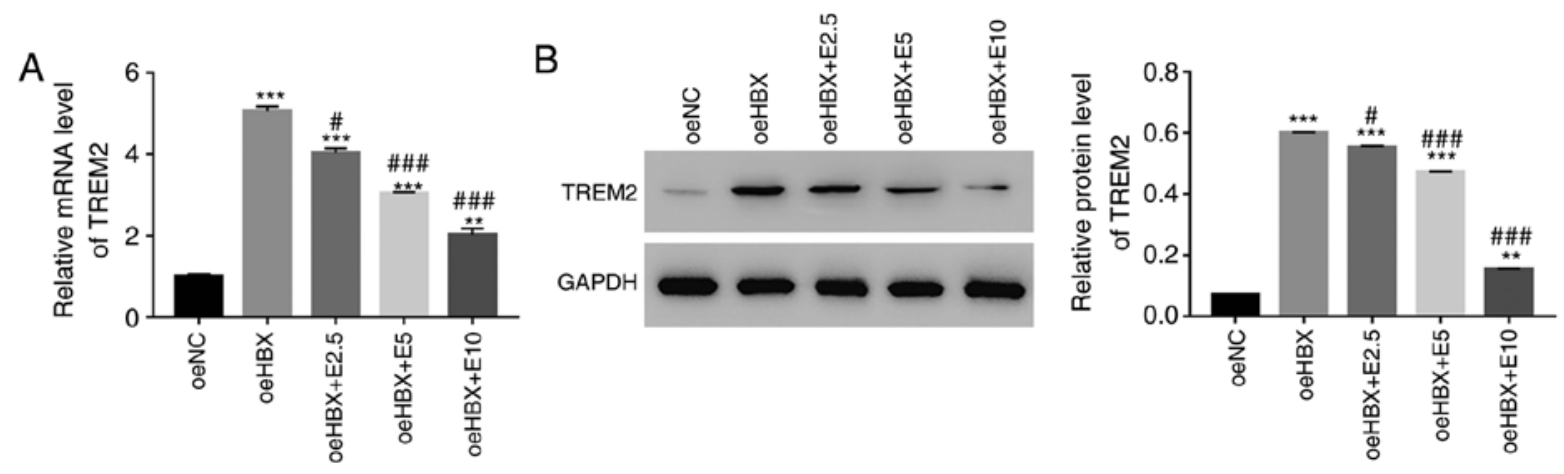

Figure 2. ECH suppresses the expression of TREM2 in oeHBX cells. (A) Relative mRNA expression levels of TREM2 were reduced by ECH in oeHBX cells. (B) Relative protein expression levels of TREM2 were reduced by ECH in oeHBX cells. ${ }^{* *} \mathrm{P}<0.01$ and ${ }^{* * * *} \mathrm{P}<0.001$ vs. oeNC. ${ }^{\#} \mathrm{P}<0.05$ and ${ }^{\# \# \#} \mathrm{P}<0.001$ vs. oeHBX. ECH, echinacoside; TREM2, triggering receptor expressed on myeloid cells 2; oe, overexpression; HBX, hepatitis B virus X; NC, negative control; E1, 1 mg/l ECH; E2.5, 2.5 mg/l ECH; E5, 5 mg/l ECH; E10, 10 mg/l ECH; E20, 20 mg/l ECH; E50, 50 mg/l ECH.
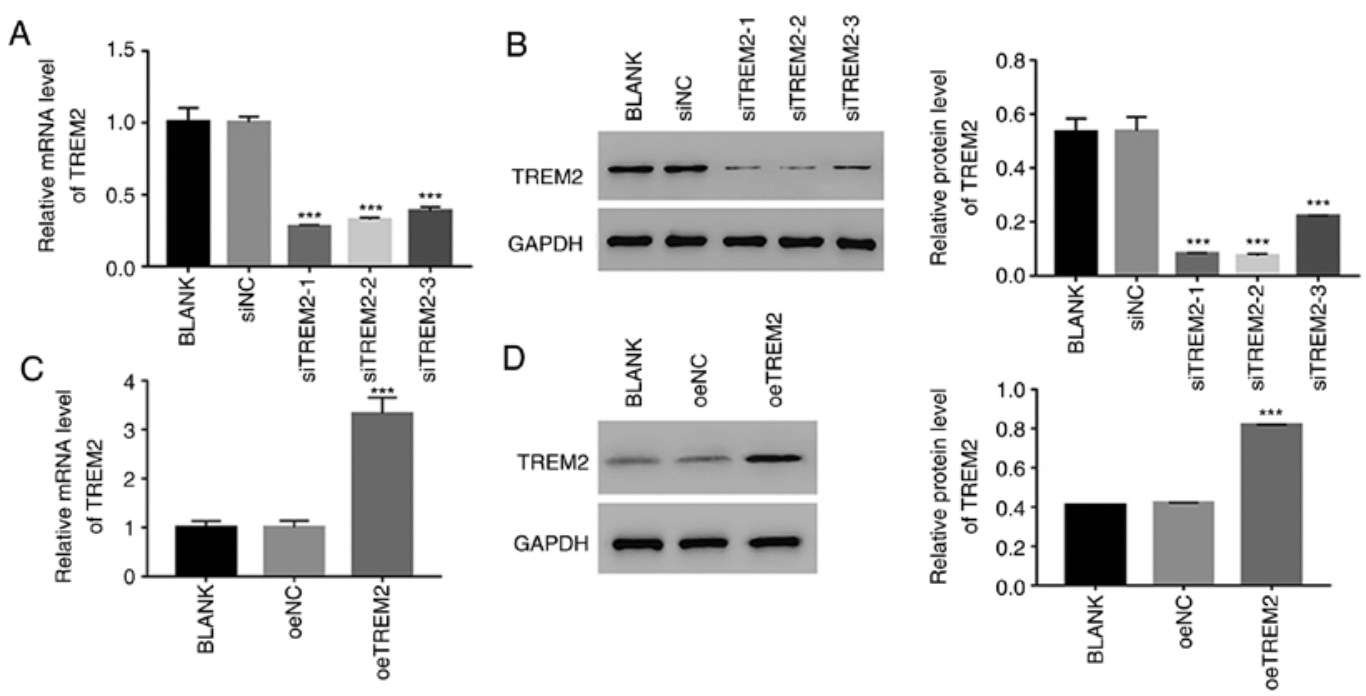

Figure 3. Knockdown and overexpression of TREM2 in HK-2 cells. Relative (A) mRNA and (B) protein expression levels of TREM2 were suppressed by siTREM2 RNAs. ${ }^{* * * *} \mathrm{P}<0.001$ vs. siNC. Relative (C) mRNA and (D) protein expression levels of TREM2 in HK-2 cells overexpressing TREM2. ${ }^{* * * *} \mathrm{P}<0.001$ vs. oeNC. TREM2, triggering receptor expressed on myeloid cells 2; si, small interfering RNA; oe, overexpression; NC, negative control.

mock plasmid served as a negative control (oeNC). The level of TREM2 expression was significantly increased in oeTREM2 cells compared with oeNC cells (Fig. 3C and D). Therefore, oeTREM2 cells were used for subsequent experimentation.

TREM2 silencing decreases the apoptosis of oeHBX cells. The apoptosis profile of oeHBX cells transfected with siTREM2 or siNC was assessed. The apoptosis rate of oeHBX + siNC cells was significantly higher compared with oeNC cells. However, the apoptosis rate was significantly decreased in oeHBX + siTREM2-1 or siTREM2-2 cells compared with oeHBX + siNC cells (Fig. 4A). The results suggested that TREM2 knockdown inhibited the function of HBX during HK-2 cell apoptosis.

Survivin belongs to the inhibitor of apoptosis protein family, which inhibits apoptotic activity and suppresses cell death (25). Targeting Survivin has been identified as a novel approach for the treatment of renal cell carcinoma (26). NF- $\mathrm{kB}$ is also an apoptosis inhibitor that plays a role in antiapoptotic tumor processes (27). Furthermore, caspase 3 activation is closely associated with apoptosis (28). In the present study, the protein content of TREM2, Survivin and cleaved caspase 3 in HK-2 cells was quantified. The level of TREM2 expression was significantly decreased in oeHBX + siTREM2-1/2 cells compared with oeHBX + siNC cells (Fig. 4B). The level of Survivin expression was significantly increased in oeHBX + siTREM2-1/2 cells compared with oeHBX cells. Additionally, the level of cleaved caspase 3 expression was significantly decreased in oeHBX + siTREM2-1/2 cells compared with oeHBX + siNC cells (Fig. 4B). Furthermore, oeHBX significantly decreased the translocation of NF- $\mathrm{KB}$ to the nucleus, and siTREM2-1/2 transfection significantly increased NF- $\kappa \mathrm{B}$ nuclear translocation (Fig. 4B). Taken together, the results suggested that TREM2 was a downstream factor of HBX in HK-2 cells, therefore, HBX might promote apoptosis by regulating TREM 2 expression in human RTECs.

TREM2 overexpression suppresses the translocation of $N F-\kappa B$ to the nucleus in $H K-2$ cells. The effect of ECH on oeTREM2-transfected cells was analysed. TREM2 overexpression promoted the apoptosis of HK-2 cells (Fig. 5A). The apoptosis of oeTREM 2 cells was significantly decreased by 

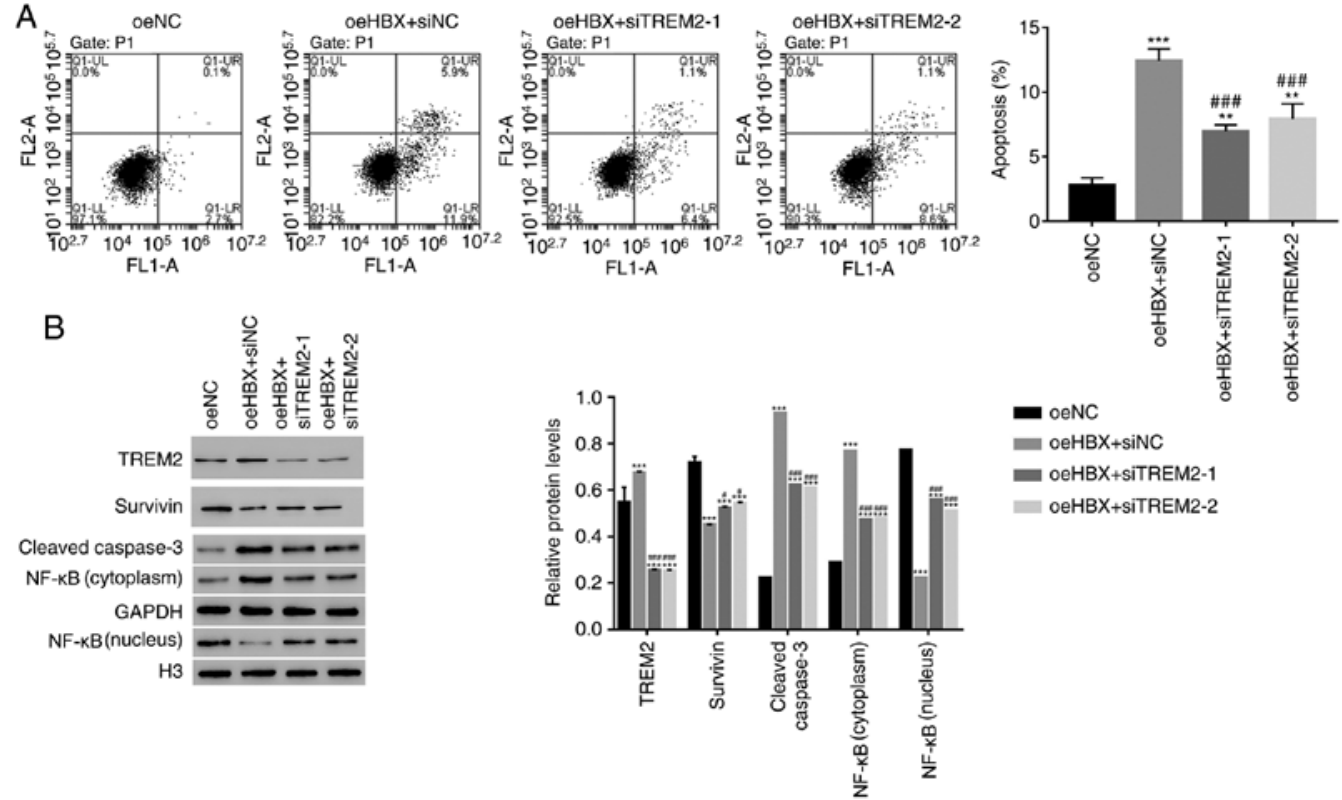

oeNC

oeHBX+sinC

- oeHBX+SITREM2-1

oeHBX+SiTREM2-2

Figure 4. HBX targets TREM2 in HK-2 cells. (A) Apoptosis rate of oeHBX cells was reduced by siTREM2-1 and siTREM2-2. (B) Western blot analysis was used to examine the protein expression levels of TREM2, Survivin, cleaved caspase3 and NF- $\kappa B$ in the different cells. ${ }^{* *} \mathrm{P}<0.01$ and ${ }^{* * *} \mathrm{P}<0.001 \mathrm{vs}$. oeNC. ${ }^{\#} \mathrm{P}<0.05$ and ${ }^{\# \# \#} \mathrm{P}<0.001$ vs. oeHBX + siNC. HBX, hepatitis B virus $\mathrm{X}$; TREM2, triggering receptor expressed on myeloid cells 2 ; oe, overexpression; si, small interfering RNA; $\mathrm{NC}$, negative control; $\mathrm{H} 3$, histone $\mathrm{H} 3$.
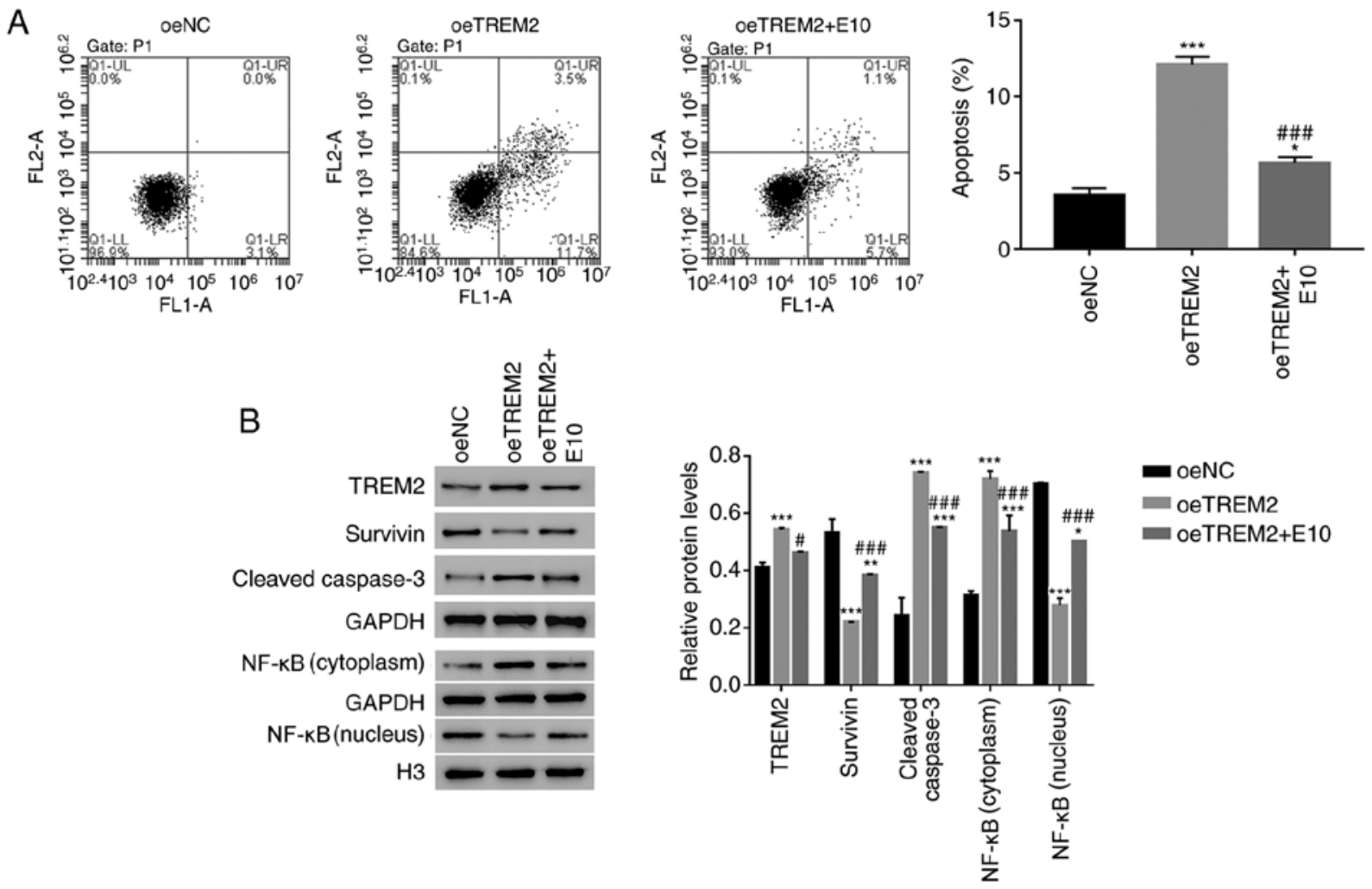

Figure 5. Overexpression of TREM2 reduces the translocation of NF- $\mathrm{KB}$ to the nucleus in HK-2 cells. (A) ECH reduced the apoptosis rate of oeTREM2 cells (B) Western blotting was used to examine the protein expression levels of TREM2, Survivin, cleaved caspase 3 and NF- $\mathrm{KB}$ in the different cells. ${ }^{*} \mathrm{P}<0.05$ and ${ }^{* * * *} \mathrm{P}<0.001$ vs. oeNC. ${ }^{*} \mathrm{P}<0.05$ and ${ }^{\# \# \#} \mathrm{P}<0.001$ vs. oeTREM2 + siNC. TREM2, triggering receptor expressed on myeloid cells $2 ; \mathrm{ECH}$, echinacoside; oe, overexpression; $\mathrm{NC}$ negative control; E10, $10 \mathrm{mg} / \mathrm{l} \mathrm{ECH}$.

ECH treatment (E10; Fig. 5A). Furthermore, ECH significantly decreased the expression of TREM2 in oeTREM2 cells. TREM2 overexpression significantly decreased the expres- sion levels of Survivin, however, this decrease in expression was reversed by ECH. Additionally, the protein expression levels of cleaved caspase 3 were significantly increased in 
A



FL1-A

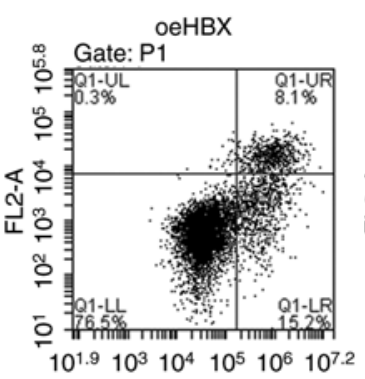

FL1-A

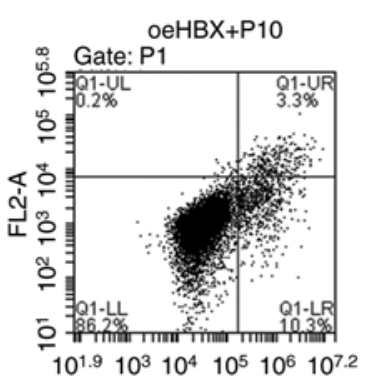

FL1-A
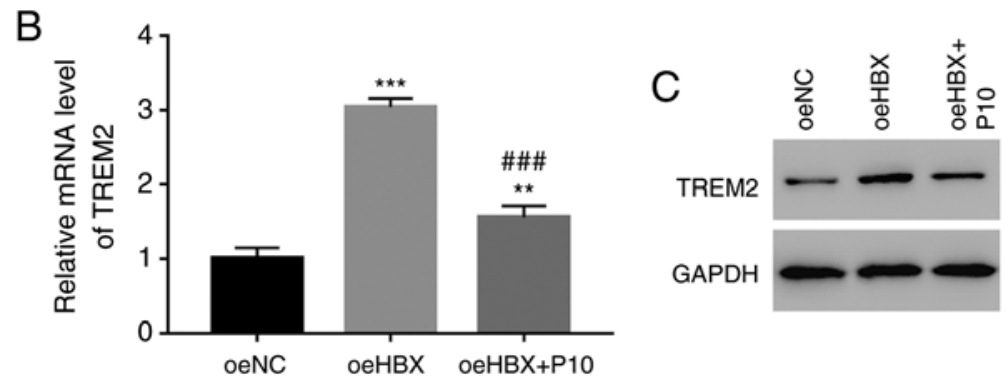

Figure 6. HBX function is suppressed by the NF- $\kappa$ B inhibitor PDTC in HK-2 cells. (A) NF- $\kappa$ B inhibitor PDTC increased the apoptosis rate of oeHBX cells. Relative (B) mRNA and (C) protein expression levels of TREM2 were decreased by PDTC in oeHBX cells. ${ }^{* *} \mathrm{P}<0.01$ and ${ }^{* * *} \mathrm{P}<0.001$ vs. oeNC. ${ }^{\# \# \#} \mathrm{P}<0.001$ vs. oeHBX. HBX, hepatitis B virus X; oe, overexpression; TREM2, triggering receptor expressed on myeloid cells 2 ; NC, negative control; P10, $10 \mu \mathrm{M}$ PDTC.

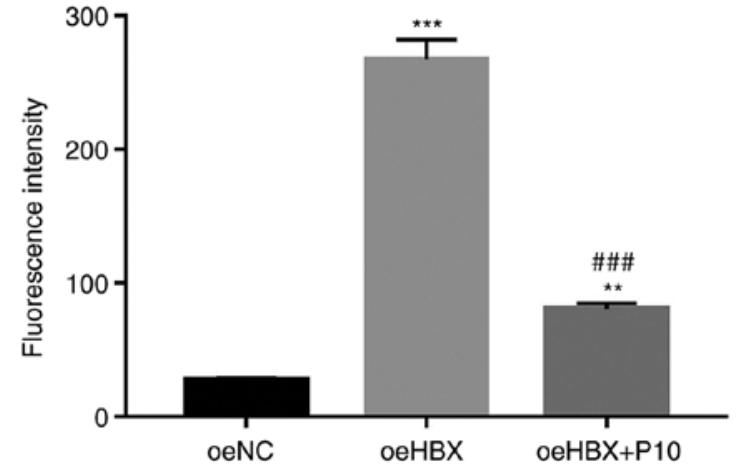

Figure 7. TREM2 promoter activity is suppressed by the NF- $\kappa \mathrm{B}$ inhibitor PDTC in oeHBX cells. ${ }^{* *} \mathrm{P}<0.01$ and ${ }^{* * *} \mathrm{P}<0.001$ vs. oeNC. ${ }^{\# \#} \mathrm{P}<0.001$ vs. oeHBX. TREM2, triggering receptor expressed on myeloid cells 2; oe, overexpression; HBX, hepatitis B virus X; NC, negative control; $\mathrm{P} 10,10 \mu \mathrm{M}$ PDTC.

oeTREM2 cells compared with oeNC cells; an effect which was significantly decreased by ECH treatment. Furthermore, $\mathrm{ECH}$ promoted the translocation of $\mathrm{NF}-\kappa \mathrm{B}$ to the nucleus in oeTREM2 cells (Fig. 5B). Taken together, the results indicated that ECH also suppressed the function of TREM2 in human RTEC cells.

$H B X$ functions are suppressed by the $N F-\kappa B$ inhibitor PDTC in human RTECs. To further assess the relationship between $\mathrm{HBX}$ and NF- $\kappa \mathrm{B}, \mathrm{HK}-2$ cells were cultured with a specific $\mathrm{NF}-\kappa \mathrm{B}$ inhibitor, PDTC $(10 \mu \mathrm{mol} / \mathrm{l}$; P10). The cell apoptosis rate of oeHBX cells was significantly increased compared with oeNC cells (Fig. 6A). However, oeHBX + PDTC cells displayed a significantly increased apoptosis rate compared with oeHBX cells. Furthermore, the relative mRNA and protein expression levels of TREM2 were decreased in oeHBX + PDTC cells compared with oeHBX cells (Fig. 6B). Therefore, the results suggested that $\mathrm{NF}-\kappa \mathrm{B}$ negatively regulated TREM2 expression in oeHBX-transfected cells.

$N F-\kappa B$ inhibitor PDTC suppresses TREM2 promoter activity in oeHBX cells. A luciferase reporter (pGL3-Enhancer-luc2) containing the wild-type promoter sequence of TREM2 (pGL3-Enhancer-luc2-pTREM2) was constructed and subsequently transfected into oeNC, oeHBX and oeHBX + P10 cultured cells.

The luciferase activity of the reporter vector containing the wild-type TREM 2 promoter was significantly increased in oeHBX cells compared with oeNC cells. However, PDTC significantly suppressed the luciferase activity of the reporter in oeHBX cells (Fig. 7). Overall, the results indicated that PDTC inhibited the transcription of TREM2 by suppressing the promoter activity of TREM 2 in oeHBX cells.

\section{Discussion}

HBX is primarily expressed in RTECs of patients with HBV-GN, and functions as a determinant of viral pathogenesis $(11,29)$. Therefore, increasing knowledge of the function of the HBX molecule network is a critical step in developing a novel approach for the treatment of HBV-GN. The aim of the present study was to further examine the function of HBX and to explore its potential molecular network in human RTECs.

HBX inhibits the proliferation of RTECs (11). In the present study, antiproliferation and pro-apoptosis functions of HBX in human RTECs were identified, suggesting that HBX suppressed the development of human RTECs.

A previous study indicated that ECH inhibits HBV replication and antigen expression (19). In the present study, $\mathrm{ECH}$ treatment disrupted the function of HBX in HK-2 cells, therefore, HBX may be affected by ECH in HK-2 cells. 
Furthermore, the results suggested that $\mathrm{ECH}$ may serve as a potential therapeutic agent for HBV-GN.

TREM2 is an innate immune receptor that plays a role in the inflammatory response (30). In the present study, HBX expression was positively associated with TREM2 expression in human RTECs. Moreover, TREM 2 knockdown significantly decreased the apoptosis rate of oeHBX cells, and ECH treatment reduced the effect of oeTREM2 on cell apoptosis. Taken together, these results suggested that TREM2 was targeted by HBX in HK-2 cells. Therefore, ECH may inhibit the apoptosis of human RTECs by blocking the activity of the HBX/TREM2 signalling pathway.

A previous report demonstrated that $\mathrm{NF}-\kappa \mathrm{B}$, not only plays a role in cell apoptosis, but is also involved in the regulation of immune responses and inflammation (31). Furthermore, TREM2 is mediated by the NF- $\kappa \mathrm{B}$-sensitive microRNA-34a during Alzheimer's disease and macular degeneration $(32,33)$. In the present study, the apoptotic rate of oeHBX cells was significantly increased in the presence of PDTC. To the best of our knowledge, the present study suggested for the first time that, the NF- $\kappa \mathrm{B}$ inhibitor PDTC suppressed the promoter activity of TREM2. Furthermore, the results suggested that TREM 2 negatively regulated the translocation of $\mathrm{NF}-\kappa \mathrm{B}$ to the nucleus in $\mathrm{HK}-2$ cells, but was positively associated with cleaved caspase 3 expression levels. TREM2 played an opposite role in HK-2 cells to its role in human degenerative nucleus pulposus and glioma cells $(22,23)$. Therefore, the present study suggested that TREM2 might have different functions in different types of human cells.

Taken together, the present study, not only indicated that $\mathrm{NF}-\kappa \mathrm{B}$ was a novel component of the HBX/TREM2 signalling pathway, but also suggested that $\mathrm{NF}-\kappa \mathrm{B}$ negatively regulates TREM2 expression in human RTECs. However, the major limitations of the present study were the lack of in vivo experiments and clinical data. Therefore, further in vivo and clinical studies are required to confirm the findings of the present study.

In the present study, the function of ECH was investigated and the results suggested the potential effects of $\mathrm{ECH}$ on the signalling pathways in human RTECs. Furthermore, the present study enhanced the existing knowledge of the biological function of ECH in human RTEC cells and also suggested its potential as a novel therapeutic agent for HBV-GN.

\section{Acknowledgements}

Not applicable.

\section{Funding}

This research was supported by the Backbone Training Project of Traditional Chinese Medicine in Yangpu District, Shanghai (grant no. YP18ZY03).

\section{Availability of data and materials}

The datasets used and/or analyzed during the current study are available from the corresponding author on reasonable request.

\section{Authors' contributions}

DG designed this project and wrote the manuscript; YZ and QW performed the experiments; LZ analyzed the data and edited diagrams. LW provided technical assistance. All authors reviewed and approved the final manuscript.

\section{Ethics approval and consent to participate}

This research was approved by the ethics committee of Yangpu District KongJiang Hospital.

\section{Patient consent for publication}

Not applicable.

\section{Competing interests}

The authors declare that they have no competing interests.

\section{References}

1. Karayiannis P: Hepatitis B virus: virology, molecular biology, life cycle and intrahepatic spread. Hepatology international 11: $1-9,2017$.

2. Seeger C and Mason WS: Hepatitis B virus biology. Microbiol Mol Biol Rev 64: 51-68, 2000.

3. Usama E, Ana Maria S, W Ray K and Fervenza FC: Treatment of hepatitis B virus-associated nephropathy. Nephron Clin Pract 119: c41-c49, 2011.

4. Zhang Y, Li J, Peng W, Yu G, Wang L, Chen J and Zheng F: HBV-Associated Postinfectious Acute Glomerulonephritis: A Report of 10 Cases. PloS one 11: e0160626, 2016.

5. Slagle BL and Bouchard MJ: Hepatitis B Virus X and Regulation of Viral Gene Expression. Cold Spring Harb Perspect Med 6: a021402, 2016.

6. Guerrieri F, Belloni L, D'Andrea D, Pediconi N, Le Pera L, Testoni B, Scisciani C, Floriot O, Zoulim F, Tramontano A, et al: Genome-wide identification of direct $\mathrm{HBx}$ genomic targets. BMC genomics 18: 184, 2017

7. Shi T, Hua Q, Ma Z and Lv Q: Downregulation of miR-200a-3p induced by hepatitis B Virus X (HBx) Protein promotes cell proliferation and invasion in HBV-infection-associated hepatocarcinoma. Pathol Res Pract 213: 1464-1469, 2017.

8. Tian Y, Xiao X, Gong X, Peng F, Xu Y, Jiang Y and Gong G: HBx promotes cell proliferation by disturbing the cross-talk between miR-181a and PTEN. Sci Rep 7: 40089, 2017.

9. Idrissi ME, Hachem H, Koering C, Merle P, Thénoz M, Mortreux F, and Wattel E: HBx triggers either cellular senescence or cell proliferation depending on cellular phenotype. J Viral Hepat 23: 130-138, 2016.

10. Wang X, Wang L, Zhu N, Zhou Y, Gu LJ and Yuan WJ: Hepatitis $\mathrm{B}$ virus $\mathrm{X}$ protein modulates renal tubular epithelial cell-induced T-cell and macrophage responses. Immunol Cell Biol 94: 266-273, 2016.

11. He P, Zhang D, Li H, Yang X, Li D, Zhai Y, Ma L and Feng G: Hepatitis B virus $X$ protein modulates apoptosis in human renal proximal tubular epithelial cells by activating the JAK2/STAT3 signaling pathway. Int J Mol Med 31: 1017-1029, 2013.

12. Yang Y, Wang X, Zhang Y and Yuan W: Hepatitis B virus $X$ protein and proinflammatory cytokines synergize to enhance TRAIL-induced apoptosis of renal tubular cells by upregulation of DR4. Int J Biochem Cell Biol 97: 62-72, 2018.

13. He P, Zhou G, Qu D, Zhang B, Wang Y and Li D: HBx inhibits proliferation and induces apoptosis via Fas/FasL upregulation in rat renal tubular epithelial cells. J Nephrol 26: 1033, 2013.

14. Han W, Luo M, He M, Zhu Y, Zhong Y, Ding H, Hu G, Liu L, Chen Q and Lu Y: HBx gene transfection affects the cycle of primary renal tubular epithelial cells through regulating cyclin expression. Mol Med Rep 18: 1947-1954, 2018.

15. Sun GD, Li CY, Cui WP, Guo QY, Dong CQ, Zou HB, Liu SJ, Dong WP and Miao LN: Review of Herbal Traditional Chinese Medicine for the Treatment of Diabetic Nephropathy. J Diabetes Res 2016: 5749857, 2016. 
16. Li L, Wan G, Han B and Zhang Z: Echinacoside alleviated LPS-induced cell apoptosis and inflammation in rat intestine epithelial cells by inhibiting the mTOR/STAT3 pathway. Biomed Pharmacother 104: 622-628, 2018.

17. Jia Y, Guan Q, Guo Y and Du C: Echinacoside stimulates cell proliferation and prevents cell apoptosis in intestinal epithelial MODE-K cells by up-regulation of transforming growth factor- $\beta 1$ expression. J Pharmacol Sci 118: 99-108, 2012.

18. Li F, Yang Y, Zhu P, Chen W, Qi D, Shi X, Zhang C, Yang Z and Li P: Echinacoside promotes bone regeneration by increasing OPG/RANKL ratio in MC3T3-E1 cells. Fitoterapia 83: 1443-1450, 2012

19. Dai LH, Shen YM, Wu YH, Yu XP, Hu HJ, Mi YJ and Chen JJ: Effect of echinacoside on replication and antigen expression of hepatitis B virus. Zhongguo Zhong Yao Za Zhi 40: 3047, 2015 (In Chinese).

20. Cantoni C, Bollman B, Licastro D, Xie M, Mikesell R, Schmidt R, Yuede CM, Galimberti D, Olivecrona G, Klein RS, et al: TREM2 regulates microglial cell activation in response to demyelination in vivo. Acta Neuropathol 129: 429-447, 2015.

21. Ulland TK, Song WM, Huang SC, Ulrich JD, Sergushichev A, Beatty WL, Loboda AA, Zhou Y, Cairns NJ, Kambal A, et al: TREM2 Maintains Microglial Metabolic Fitness in Alzheimer's Disease. Cell 170: 649-663.e613, 2017.

22. Wang XQ, Tao BB, Li B, Wang XH, Zhang WC, Wan L, Hua XM and Li ST: Overexpression of TREM2 enhances glioma cell proliferation and invasion: a therapeutic target in human glioma. Oncotarget 7: 2354-2366, 2016.

23. Bai M, Yin HP, Zhao J, Li Y and Wu YM: Roles of TREM2 in degeneration of human nucleus pulposus cells via NF- $\mathrm{kB}$ p65. J Cell Biochem 119: 8784-8796, 2018.

24. Livak KJ and Schmittgen TD: Analysis of relative gene expression data using real-time quantitative PCR and the 2(-Delta DeltaC(T)) Method. Methods 25: 402, 2001.

25. Jaiswal PK, Goel A and Mittal RD: Survivin: A molecular biomarker in cancer. Indian J Med Res 141: 389-397, 2015.
26. Carew JS, Espitia CM, Zhao W, Mita MM, Mita AC and Nawrocki ST: Targeting survivin inhibits renal cell carcinoma progression and enhances the activity of temsirolimus. Mol Cancer Ther 14: 1404, 2015.

27. Ohshima K, Sugihara M, Haraoka S, Suzumiya J, Kanda M, Kawasaki C, Shimazaki K and Kikuchi M: Possible immortalization of Hodgkin and Reed-Sternberg cells: telomerase expression, lengthening of telomere, and inhibition of apoptosis by NF-kappaB expression. Leuk Lymphoma 41: 367-376, 2001.

28. Yang LQ, Fang DC, Wang RQ and Yang SM: Effect of NF- $\mathrm{kB}$, survivin, Bcl-2 and Caspase 3 on apoptosis ofgastric cancer cells induced by tumor necrosis factor related apoptosis inducing ligand. World J Gastroenterol 10: 22-25, 2004.

29. Bouchard MJ and Schneider RJ: The enigmatic X gene of hepatitis B virus. J Virol 78: 12725-12734, 2004.

30. Zhong L, Chen XF, Wang T, Wang Z, Liao C, Wang Z, Huang R, Wang D, Li X, Wu L, et al: Soluble TREM2 induces inflammatory responses and enhances microglial survival. J Exp Med 214: 597-607, 2017.

31. Dolcet X, Llobet D, Pallares J and Matias-Guiu X: NF- $\kappa B$ in development and progression of human cancer. Virchows Arch 446: 475-482, 2005.

32. Zhao Y, Bhattacharjee S, Jones BM, Dua P, Alexandrov PN, Hill JM and Lukiw WJ: Regulation of TREM2 expression by an NF-KB-sensitive miRNA-34a. Neuroreport 24: 318-323, 2013.

33. Bhattacharjee S, Zhao Y, Dua P, Rogaev EI and Lukiw WJ: MicroRNA-34a-mediated down-regulation of the microglial-enriched triggering receptor and phagocytosis-sensor TREM 2 in age-related macular degeneration. PLoS One 11: e0150211, 2016.

This work is licensed under a Creative Commons Attribution-NonCommercial-NoDerivatives 4.0 International (CC BY-NC-ND 4.0) License. 